\title{
Design and development of a robotic bee for the analysis of honeybee dance communication
}

\author{
T. Landgraf*, H. Moballegh and R. Rojas \\ Department of Mathematics and Computer Science, Freie Universität Berlin, Berlin, Germany
}

(Received 25 February 2008; final version received 12 November 2008)

\begin{abstract}
We have designed a robotic honeybee to mimic the bee dance communication system. To achieve this goal, a tracking system has been developed to extract real bee dance trajectories recorded with high-speed video cameras. The results have been analysed to find the essential properties required for the prototype robot. Putative signals in the dance communication have been identified from the literature. Several prototypes were built with successive addition of more features or improvement of existing components. Prototypes were tested in a populated beehive results were documented using high-speed camera recordings. A substantial innovation is a visual feedback system that helps the robot to minimise collisions with other bees.
\end{abstract}

Keywords: bee robot; honeybee dance; biomimetic robots

\section{Introduction}

In this paper we describe the design and development of a robotic honeybee capable of mimicking the honeybee dance. After 60 years of honeybee dance research, fundamental questions remain open regarding the information transfer between dancer and follower bees. Several signals and sensory modalities have been hypothesised to play an important role in the decoding process. A robot able to produce all essential signals independently from each other would allow a deeper analysis of the bee dance.

We began our research by asking how bees dance. We recorded hundreds of high-speed videos of bee dances which were processed with bee-tracking software. The resulting trajectories were analysed statistically. A realistic mechanical reproduction of the waggle behaviour was defined using these data. Having delimited the range of possible movements, we started the design process of our robotic bee. We tested several prototypes and improved our models according to the results. We identified the ability of the robot to react to nearby conditions as an essential component and therefore developed a visual feedback system that prevents the model from colliding with surrounding bees.

This paper is organised as follows: First, the bee dance behaviour is reviewed briefly, and open questions and current notions are explained. Possible signals in the dance context are briefly discussed. In the second part we give an overview of the tracking system used to obtain trajectories of bee dances and the results of such analysis. In the last part of this contribution we focus on the design principles and implementation details of the robotic bee. Finally, early experimental results and future work are discussed.

\section{The honeybee dance}

Honeybees (Apis mellifera) have always attracted attention due to their famous 'waggle dance', an intriguing and complex behaviour that bees use to convey spatial information on desirable resources. Returning forager bees can be observed performing vigorous ('dance') movements on the vertical honeycomb. The dancer bee moves forward in a rather straight line, waggling its body from side to side at a frequency of approximately $15 \mathrm{~Hz}$. Each straight waggle run is followed by alternating left and right semicircular trajectories back to the approximate starting point of the previous waggle run. The angle of the waggle run with respect to gravity gives direction to the target with respect to the sun's azimuth. The distance is encoded in the length and duration of the waggle run (Michelsen, 2003; Seeley 1995; Von Frisch 1965).

Dance communication in honeybees has often been described as one of the most impressive forms of communication in animals. However, more than 60 years after the initial discovery of the meaning of the dance (Von Frisch 1946) it remains obscure how bees manage to decode information in the dance and thus which of the many dance signals carry information. Honeybees produce a variety of stimuli. When waggling to and fro the dancer bee is likely to touch the nearby followers (Rohrseitz and Tautz 1999). High-speed videos reveal many contacts of the waggling dancer with the head and antennae of the surrounding follower bees. The number of antennal deflections of a follower bee is highly correlated with the number of waggling movements (De Marco 2007). When performing the waggle run, the dancer also produces bursts of $280-\mathrm{Hz}$ wing oscillations. Such

\footnotetext{
*Corresponding author. Email: landgraf@mi.fu-berlin.de
} 
oscillations produce slight airflows at the rims of the wings to maintain pressure balance (Esch et al. 1965; Michelsen 1986; Wenner 1962). Follower bees might be able to detect the airflows (Tsuijuchi 2007). Furthermore long continuous airflows ('jets') described by Storm (2002) and Michelsen (2003) are generated by the wing oscillations and might also be detectable by follower bees standing behind the dancer. Warnke (1976) described the production of electrical fields by the wing movement, whose detection has not been shown yet. Information without a directional component, e.g. attention arousal, might be transmitted by odour chemical and semiochemical cues (Dyer 2002; Farina et al. 2005; Thom et al. 2007) or substrate-borne vibrations (Nieh and Tautz 2000; Tautz 1996; Tautz et al. 2001).

Since the dance signals (especially the signals produced by the wings) are strongly coupled one cannot observe the effect of one signal alone. A possible way of uncoupling the signals is to use a robotic bee and therewith be able to fully control and modulate the output of the sender (Haldane and Spurway 1954). Michelsen and colleagues (1992) built the first robotic bee that produced a waggle coupled with wing oscillations. The reported recruitment rate of the robotic bee was 5 to 10 times lower than that of the live dancers.

Recent work (Michelsen 2003; Thom et al. 2007) points out signals which were not integrated into Michelsen's robot. Furthermore, the importance of body contacts was not considered in Michelsen's robot. First, the mass of the bee model and the steering method had not been designed to simulate the impact force of real bee dancers' waggling impacts on follower bees. Second, Michelsen's robot was steered in a static trajectory without caring about running into nearby bees.

\section{Analysis of the bee dance via high-speed videos and an automatic tracking system}

The dancer's body movement, especially in the waggle phase, seems to be one of the most important signals to the follower bees (De Marco 2007; Rohrseitz and Tautz 1999). The waggle run might be directly (via body contacts) or indirectly (by producing spatially and temporally unique signal patterns) responsible for the emission or transmission of information. Thus the waggle must be reproduced as precisely and naturally as possible.

We developed an automatic tracking system (Landgraf and Rojas 2007) to gain data from previously recorded high-speed videos of bee dances. The tracking algorithm is based on sparse optical flow fields that are clustered by coherent motion using a Hough-transform-like method ('Motion-Hough'). Since it relies only on motion, the algorithm is robust against different lighting conditions, magnification factors, marked or unmarked bee and the like. The tracking system was used to build a database of dance trajectories. Figure 1 shows a typical bee dance trajectory. We detected automatically the waggle phases and extracted various properties such as the waggle frequency, mean and variance of the waggle direction and lateral displacement in the waggle phase and its rotational component. Interestingly the stereotypical 'figure of eight' described in the literature is hardly to be seen in real trajectories. We observed that the beginning point of a new waggle run is almost always shifted due to evasion manoeuvres of the dancer that just reacts to the crowded hive situation. We observed full stops, evasions and even backward movements which in turn are rarely seen in the waggle run. Analogously the variance of waggle run duration is much lower than the return run's. An exhaustive description of the analysis is given in Landgraf et al. (2008) which confirms the results obtained from manual measurements and augments the analysis of De Marco et al. (2008).

The high-speed recordings have also been analysed to gain knowledge about collision prevention in real bees. A dancer bee hits other bees mostly with the head and antennae. A dancer can often hit other bees in return runs with the sides of the body. Also, we observed that bees seem to ignore slight collisions but move back when hit harder with force above a certain threshold. In the dance context, we did observe following kinds of collisions:

(1) Waggle collisions. The dancer hits other bees when performing the waggle run. The followers are hit frontally against the antennas and the head by the dancer's abdomen, or the dancer runs into another bee frontally.

(2) Return run collisions. The dancer runs into a follower or uninvolved bee with the head, or while turning the dancer hits another nearby bee with the sides.

(3) Follower collisions. A follower searching for the dancer runs into her or other followers.

(4) Pass-by collisions. Bees passing by the dance area hit others with the head.

What all have in common is that the energy of the collision is so small that no rebound can be observed. The dancer is observed numerous times to draw aside in return run situations but conversely does not try to change the waggle direction for avoidance of collisions.

\section{Design of the robot}

In this section we report on design principles and implementation details. The prototypes to be built have to comply with these requirements:

- Portability. The whole system has to be portable, easy to install and tunable over the surface of the comb.

- Actuators. Actuators must not generate acoustical, mechanical or electromagnetic noise near the bees (i.e. only within reasonable ranges). 

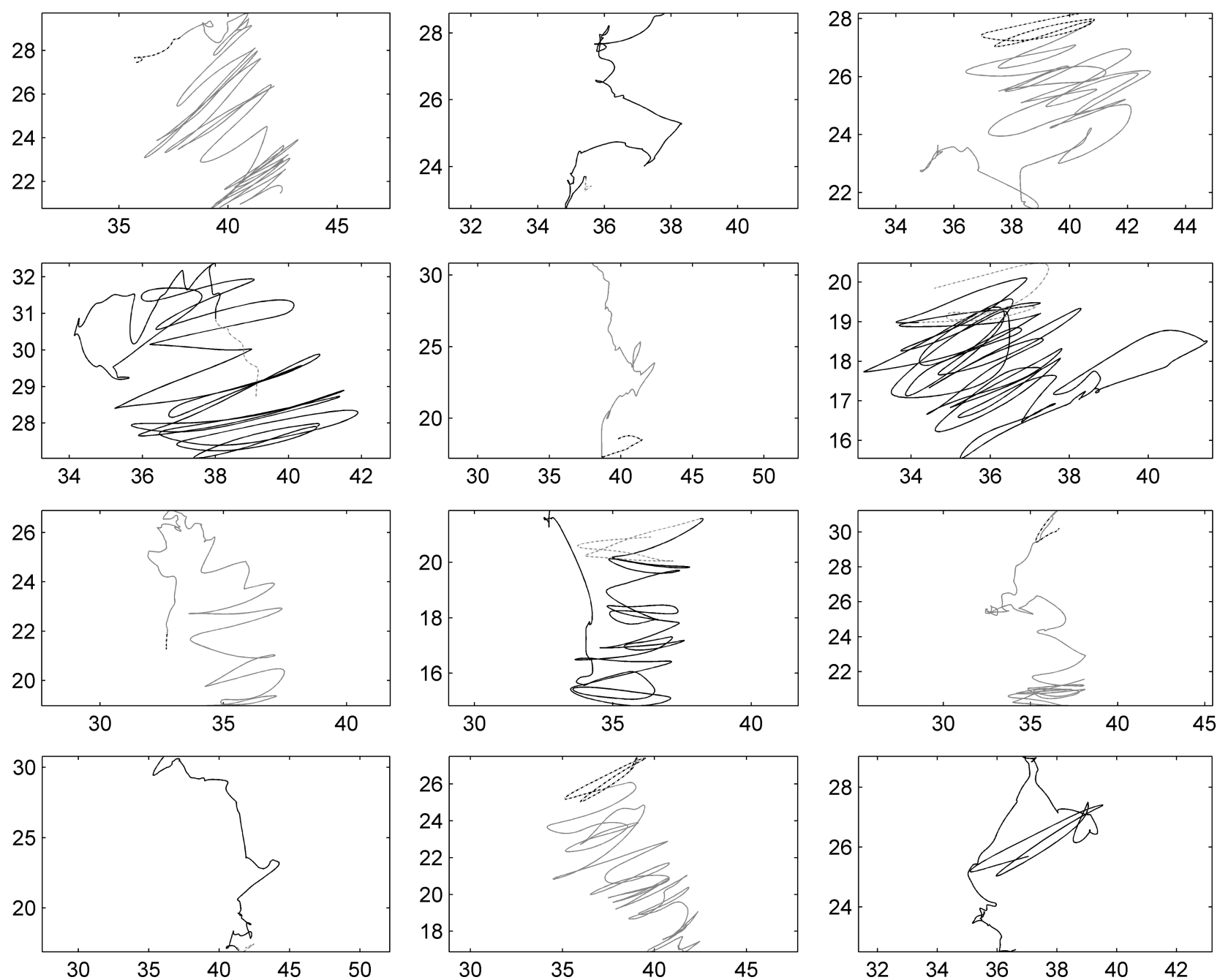

Figure 1. The trajectory of a bee dance obtained with the tracking system. Only the positions of the centre of the bee are plotted throughout the waggle dance. For the sake of clarity the whole trajectory is split into non-overlapping parts ordered from left to right and top to bottom. Grey and black colours are alternating. In every subplot $10 \%$ of the following trajectory is drawn as a dashed line in a different colour. Waggle phases are articulated and followed by alternating left and right return runs. Measurements are in mm.

- Physical properties. The body mass, material and shape of the artificial bee must have realistic values. The movement should induce comparable forces into other bees.

- Signals. All types of stimuli generated by real bee dancers must be produced and controlled individually by the robot bee.

- Movements. The movements of live dancers are highly dynamic and strongly coupled with the amount of followers in the near field, the amount of free or blocked space in the projected dance path and other highly unforeseeable and dynamic variables. Thus the dance pattern has to be modelled parametrically as a closed feedback system, using sensors to recognise obstacles.

In order to achieve these goals, several design and control ideas have been implemented and will be presented in the remainder of this section.

\section{Hardware framework and low-level control}

For the connection of the actuators and signal units we have customised the well-known bioloid framework. It is a commercially available robotic kit that we use as our hardware basis. Extensions to the robot design can be easily integrated into the framework using numerously available bioloid compatible components. The main control unit, the CM5, is a versatile utilisable IO module that wraps an Atmel Atmega 128 microcontroller. The CM5 can be programmed using C. Communication to servo and signal units and also to a mobile PC is done via standard RS232 protocol. The CM5 is used to generate all low-level behaviours like the waggle movement (see below), whereas the mobile PC covers the heavy computations and the user interface (Figure 2). The PC is also connected to two high-speed cameras for the recognition of obstacles. 


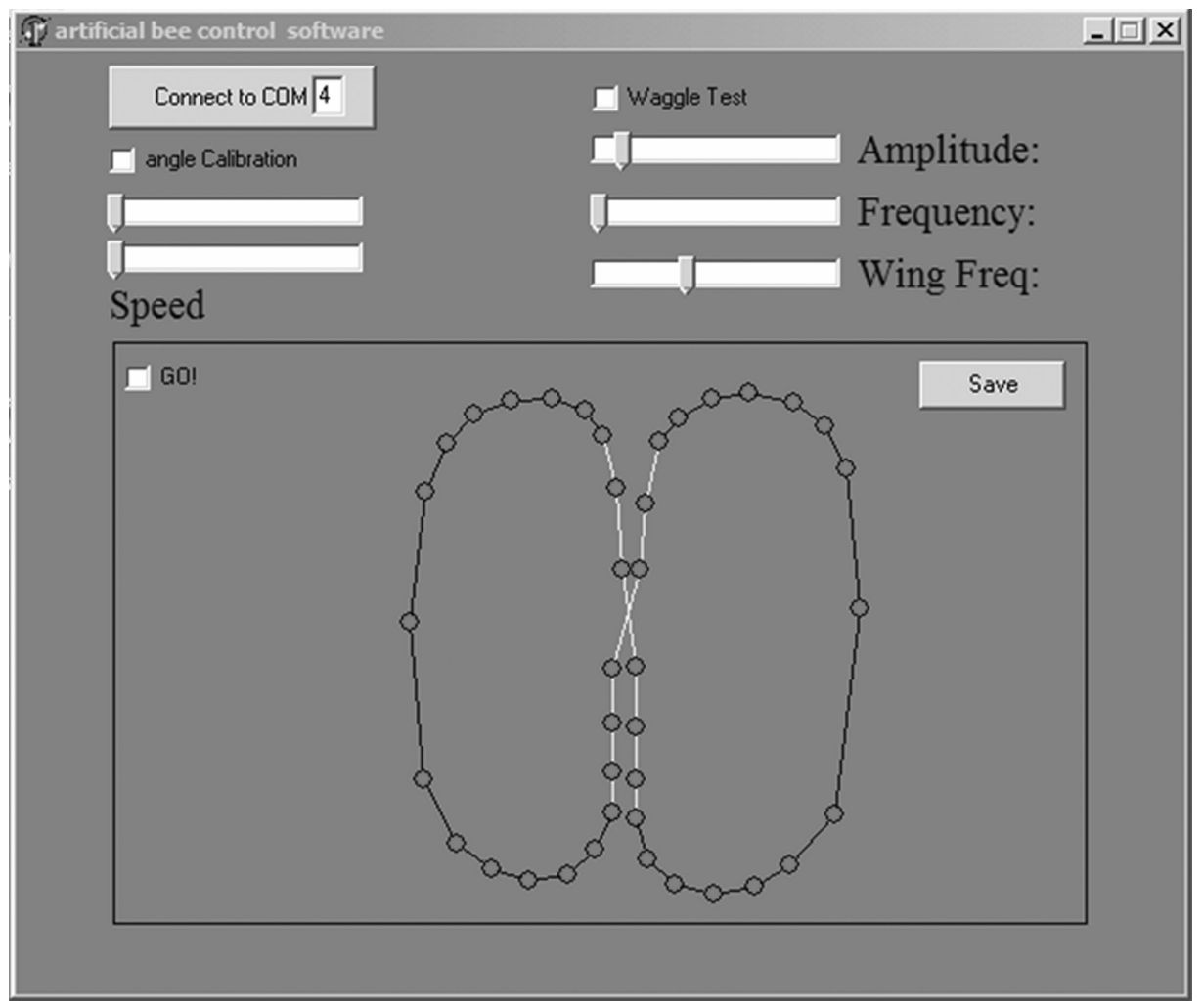

Figure 2. User interface of the robot control program. Static waggle trajectory can be defined via simple mouse input. Coordinates are sent via RS232 protocol to robot control module and translated there into motor commands.

In experimental trials we use another PC with a third camera that helps to set up the robot as well as give the basis for an 'offline' analysis of robot-to-follower interactions.

\section{Motion mechanism}

The robotic bee must be able to move in a two-dimensional Cartesian coordinate system, rotate and vibrate the body based on the previously described parameters. In addition, the robot should be compact and portable and easy to install on the hive. To achieve this goal and also maximise the distance between actuators and the bees we have taken advantage of a parallel motion mechanism. All of the actuators are fixed to a frame and apply their forces/torques to a single point. This is contrary to the common motion mechanisms like plotters or computer numerical control (CNC) machines, in which the actuators of each axis carry the next stage as used by Michelsen (1992). Parallel motion mechanisms have several advantages such as simplicity and lower power consumption as well as some disadvantages like high degrees of non-linearity and the requirement of high DOF joints to connect all actuators at one point. The problem of non-linearity can be solved in two ways, first by using the inverse kinematics in the control software and second by using the mechanism in a relatively small neighbourhood in which the non-linearity is negligible. In the design of the motion mechanism for the robot both of the solutions are used (see Figures 3 and 4).

The motion mechanism of the current bee robot prototype consists of three actuators; two servo motors that produce the two-dimensional movement on the surface of the hive and one servo motor that generates the rotation. For the waggle motion all three servo motors cooperate to produce the lateral displacements of about one bee width ( $\sim 6 \mathrm{~mm}$ ) and the rotational component of up to $45^{\circ}$ for one waggle period.

\section{Signals}

The most important signal might be body contacts. Therefore the shape, surface and motion of the dummy bee used on the end of the rod have to be perfectly reproduced. To accomplish the former two we also use caught live dancers. They are collected using a small customised vacuum cleaner that holds ice packs in its interior. The captured bee dies within 2-5 minutes and can be used for approximately 1 hour before the other bees react in a disturbed manner. The bee is glued onto the robot terminal. The waggle movement is generated on the CM5. High-speed video recordings show a highly realistic wagging motion. 

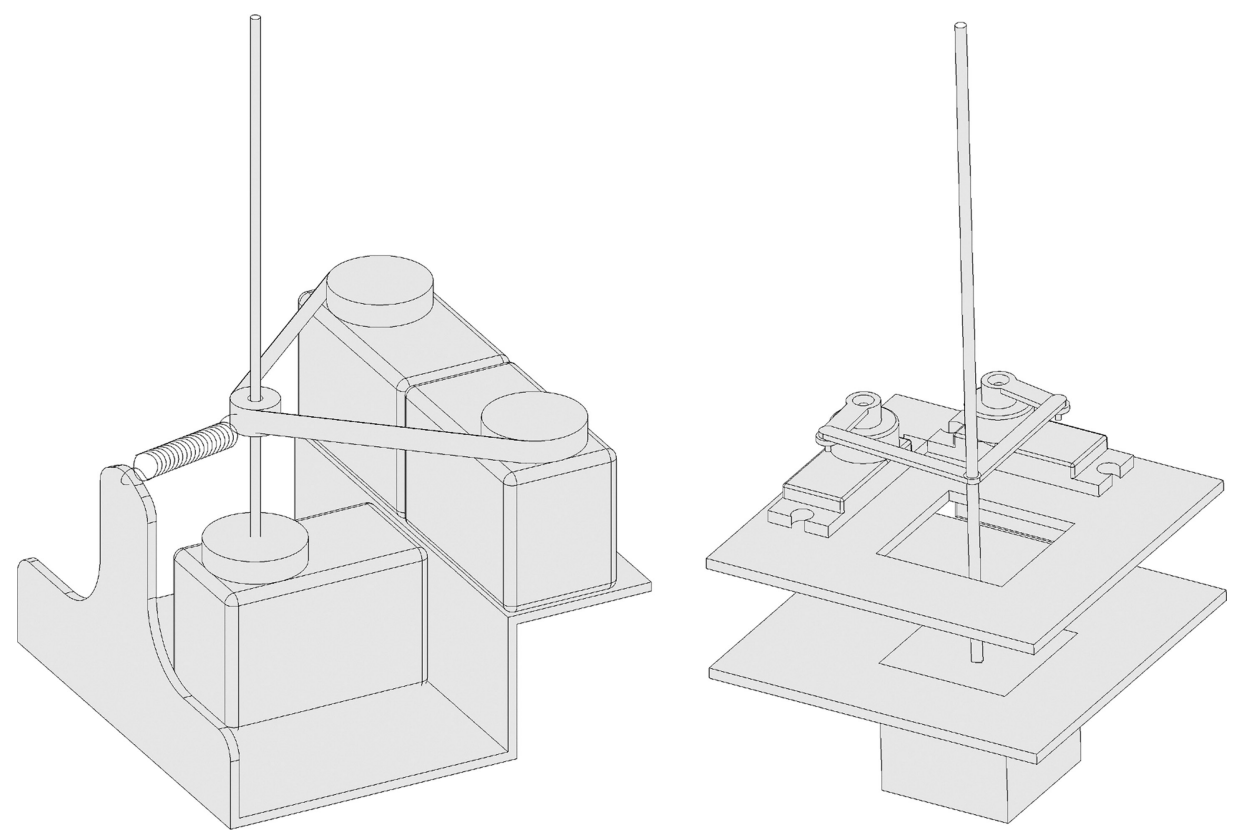

Figure 3. Computer-aided drawing (CAD) models of the prototypes. In the first prototypes (right) we used a stepper motor to control the orientation of the bee model. This was changed because the stepper motion was not smooth enough - the model vibrated too much. The new current design principle is depicted in the left figure. The rotational component is now driven by a servo motor.

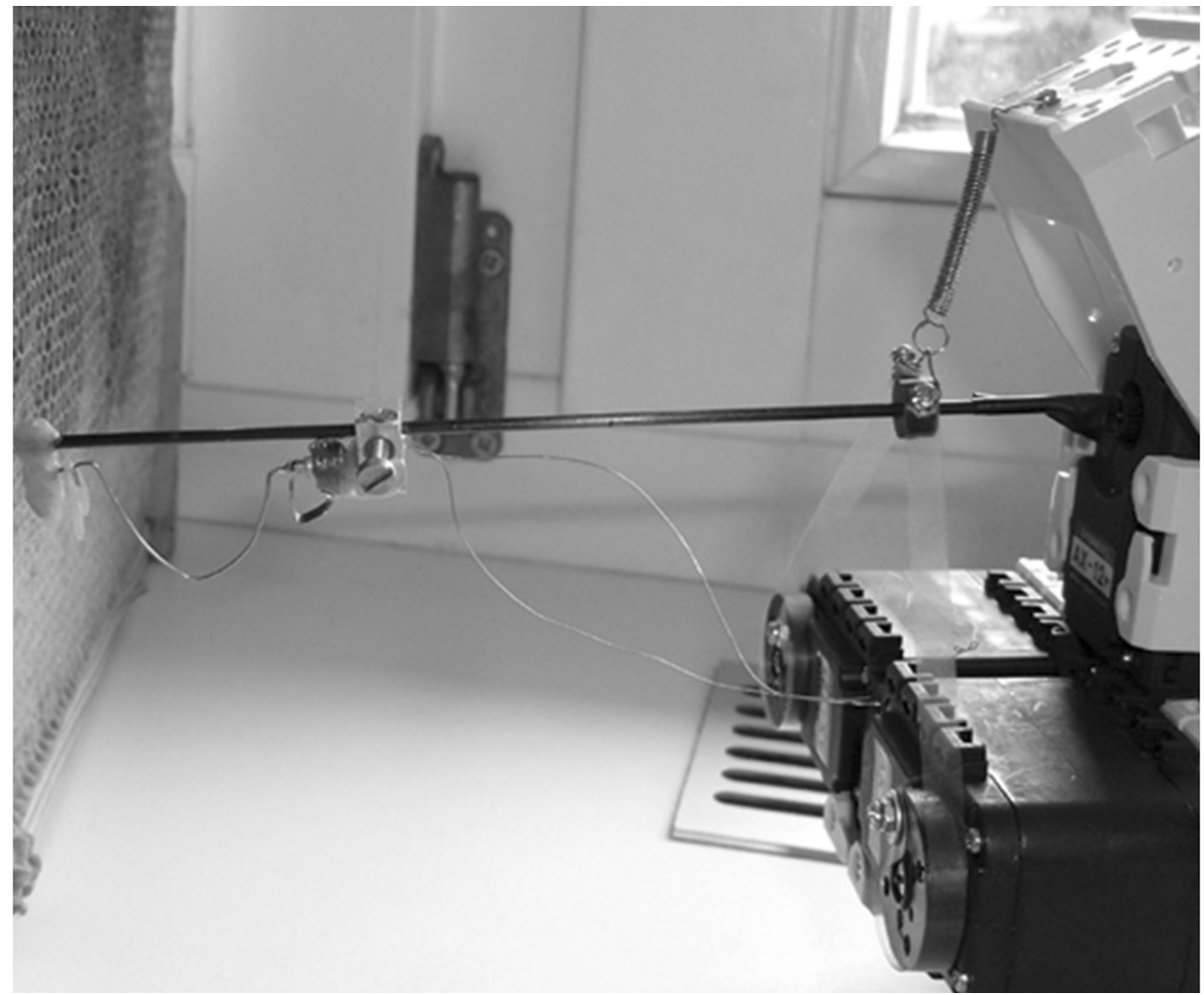

Figure 4. The current prototype with wing motion mechanism. The solenoid generates a small vibration of a magnet, which is amplified by the resonator. The excitation frequency can be an integer fraction of the resonance frequency of the resonator. 


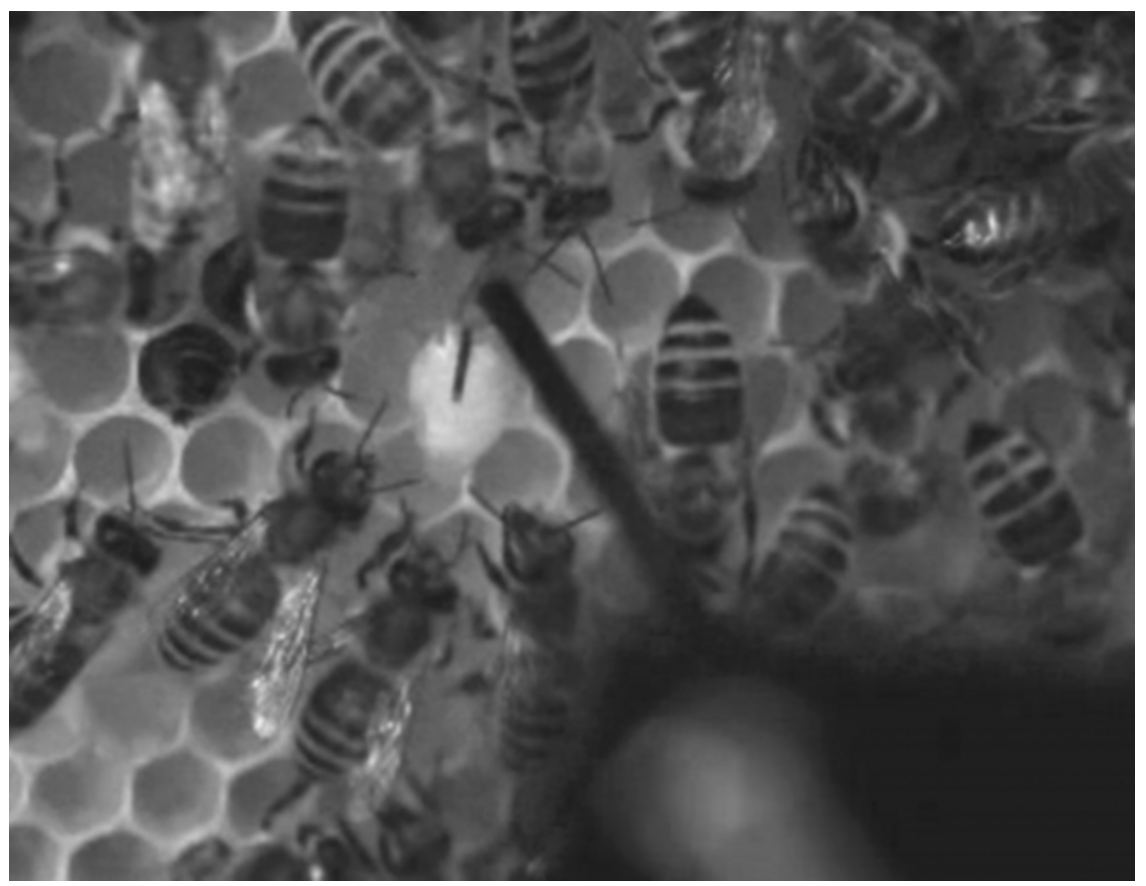

Figure 5. A test trial using the first prototypes without wing vibration unit. The bee model is held into the open hive and moved according to a waggle dance path. Three bees at the back of the robotic model are approaching.

Any artificial bee model has to exhibit a certain smell that camouflages the object as originating from the hive being used. Using a bee model made of beeswax that was put into the hive several days before the experiments generated the smallest amount of disturbance.

Live bee dancers vibrate their wings in bursts of about $15-$ millisecond length of a $280-\mathrm{Hz}$ carrier with a repetition rate of about $28 \mathrm{~Hz}$ (Michelsen, 1986). This higher frequency component cannot be produced by the muscles directly. In fact the bees use the resonance frequency of the thorax to produce this vibration, using a much lower frequency. The design of the wing actuation system faces the same problem because it is not easy to produce a relatively high amplitude and frequency in the wings without using a powerful actuator which would be heavy enough to disturb the movement of the robot. A solution similar to that of the bees is used in the design of the wing mechanism of the robot. A small solenoid generates the vibration with the same or an integer fraction of the target frequency. This vibration is then passed through a mechanical resonator which has enough different resonance frequencies along each axis. The resonator can be adjusted to amplify the vibration only in the dorsoventral direction. The wings are installed at the other end of the resonator. High-speed video recordings show the pulsed oscillations at the target frequency of $280 \mathrm{~Hz}$. In compliance with the fourth requirement this wing oscillator produces by design no jet airflows but only the wanted oscillating currents at the rims of the wing plate.

\section{Reactive vision}

As described earlier, the waggle motion has to be dynamically generated using some kind of sensor to recognise objects blocking the next few millimeters the robot wants to move in. In experimental trials with the robot prototypes we identified the return runs as the most perturbing part of the dance. The deflection of the abdominal part of the robot caused many collisions. Reducing the overall speed of motion the number of severe collisions was decreased but not prevented.

We have tested different methods for recognising obstacles (other bees) using high-speed cameras. Because of the high frame rates needed for the recognition we favour a very simple method. We illuminate the comb surface from the back side and - in the camera images - apply a simple threshold to separate the bees from the background. This method is very robust and fast but needs a lot of preparation of the hive. All bees have to be removed from the back side and furthermore prevented from changing sides again. Currently we are building a new observation hive with special specifications. The vision system is implemented as a closed feedback system. Video frames are queried from a FireWire camera and saved into a ring buffer. The recognition thread polls a new frame and finds regions the robot will pass through and which are (or in near future will be) occupied by other bees. These regions are marked in the 'threat map'. The path-planning routine reads the current trajectory and threat map and alters the trajectory if necessary. 


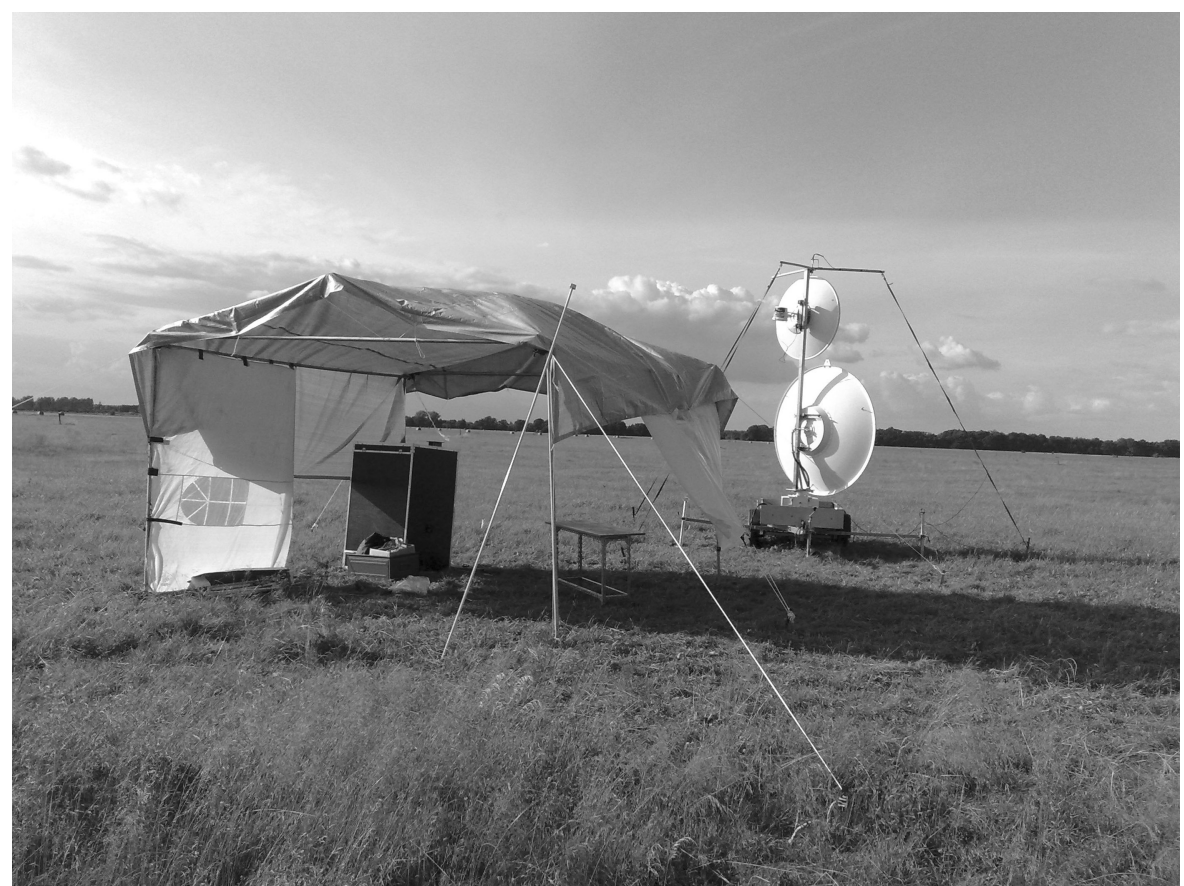

Figure 6. The radar receiver on top of the emitter's parabolic antenna currently in use for honeybee navigation experiments. (Image courtesy: Anke Schumann.)

\section{Discussion}

Although the idea of a honeybee robot is not entirely new, the project was encouraged by the emergence of new signal hypotheses as well as the computational feasibility of real-time high-speed video analysis for reactive behaviour. By applying an exhaustive video analysis and highprecision motion tracking of honeybee dances we were able to identify motion parameters and behavioural properties

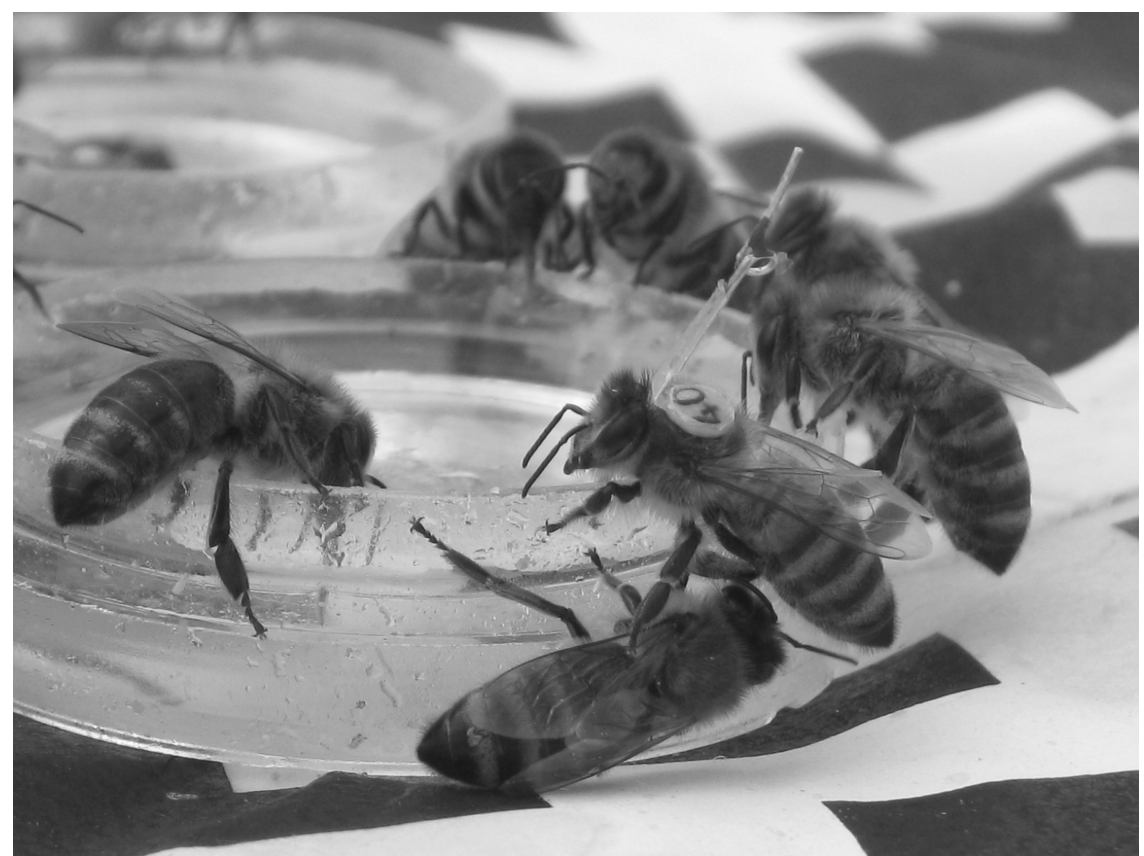

Figure 7. A forager bee equipped with a radar transponder. To be able to identify the bees uniquely, small numbered tags are glued to the bee's thorax as well. (Image courtesy: Anke Schumann.) 
of dancing honeybees. This knowledge was put into the design of new prototypes. So far, the waggle dance and wing vibrations have been successfully implemented. The robot is lightweight and highly portable. After several improvements the model bee was not attacked in experiments and even followed by other bees. However, no recruitment has been achieved yet. First experimental results (Figure 5) obtained in 2006 suggest that a reactive behaviour control might be essential for recruitment success. The robot control must be able to at least lower the magnitude of an imminent collision, if not prevent it. Therefore a reactive vision system has been implemented, which now has to be tested and tuned. The current recognition algorithm is very simple (for the sake of performance). We might need to implement more complex recognition algorithms for example to be able to extrapolate the movement of surrounding bees into the future (e.g. using optical flow methods).

In the future the variety of signals will be fully implemented. The new robot model will produce gustatory and semiochemical signals as well as jet airflows and electromagnetic fields. Also a newly designed hive will allow production of honeycomb vibrations. This future work has to assure that with the new load of actuators the movements of the robot still range within realistic parameters.

To validate the robot we plan to use a harmonic radar system provided by Professor Randolf Menzel to track the flight paths of robot-recruited bees. The system (see Figure 6) emits radar beams of a fixed frequency that are reflected harmonically by small transponders (see Figure 7) glued to the thorax of forager bees. Their positions can be tracked with a temporal and spatial resolution of about $0.3 \mathrm{~Hz}$ and $12 \mathrm{~m}$, respectively.

\section{Acknowledgements}

We are indebted to Professor Dr Randolf Menzel, Dr Rodrigo J. De Marco, Uwe Greggers and Professor Axel Michelsen for their valuable advice.

\section{References}

De Marco RJ, Gurevitz JM, Menzel R. 2008. Variability in the encoding of spatial information by dancing bees. J Exp Biol. 211:1635-1644.
De Marco R. 2007. Decoding information in the honeybee dance: re-examining the behaviour of the followers and the tactile stimuli arising from a dancer's wagging movements (personal communication).

Dyer FC. 2002. The biology of the dance language. Annu Rev Entomol. 47:917-949.

Esch H, Esch I, Kerr WE. 1965. Sound: An element common to communication of stingless bees and to dances of the honey bee. Science. 149:320-321.

Farina WM, Grüter C, Díaz PC. 2005. Social learning of floral odours inside the honeybee hive. Proc R Soc B: Biol Sci. 272:1923-1928.

Haldane J, Spurway H. 1954. A statistical analysis of communication in 'Apis mellifera' and comparison with communication in other animals. Insectes Sociaux, Springer, 1:247-283.

Landgraf T, Rojas R. 2007. Tracking honey bee dances from sparse optical flow fields. Berlin: FB Mathematik und Informatik FU. Technical Report B-07-11, 1-37.

Landgraf T, Rojas R, Menzel R, De Marco RJ. Analysis of high Precision honeybee dance trajectories (in prep) (the paper would appear in Journal of Experimental Biology in 2009).

Michelsen A, Kirchner WH, Lindauer M. 1986. Sound and vibrational signals in the dance language of the honeybee, Apis mellifera. Behav Ecol Sociobiol. 18:207-212.

Michelsen A. 2003. Signals and flexibility in the dance communication of honeybees. J Comp Physiol. 189:165-174.

Michelsen A, Andersen BB, Storm J, Kirchner WH, Lindauer M. 1992. How honeybees perceive communication dances, studied by means of a mechanical model. Behav Ecol Sociobiol. 30:143-150.

Nieh J, Tautz J. 2000. Behaviour-locked signal analysis reveals weak $200-300 \mathrm{~Hz}$ comb vibrations during the honeybee waggle dance. J Exp Biol. 203:1573-1579.

Rohrseitz K, Tautz J. 1999. Honey bee dance communication: waggle run direction coded in antennal contacts? J Comp Physiol. 184:463-470.

Seeley T. 1995. The wisdom of the hive: The social physiology of honey bee colonies. Harvard University Press, London.

Tautz, J. 1996. Honeybee waggle dance: recruitment success depends on the dancefloor. J Exp Biol. 199: 1375-1381.

Tautz J, Casas J, Sandeman D. 2001. Phase reversal of vibratory signals in honeycomb may assist dancing honeybees to attract their audience. J Exp Biol. 204:3737-3746.

Thom C, Gilley DC, Hooper J, Esch HE. 2007. The scent of the waggle dance. PLoS Biol. 5:e228.

Von Frisch K. 1946. Die Tänze der Bienen. Österr Zool Z. 1:1-48

Von Frisch K. 1965. Tanzsprache und Orientierung der Bienen. Springer-Verlag, Berlin.

Warnke U. 1976. Effects of electric charges on honeybees. Bee World. 57:50-56.

Wenner, A. 1962. Sound production during the waggle dance of the honey bee. Anim Behav, Elsevier, 10:79-95. 

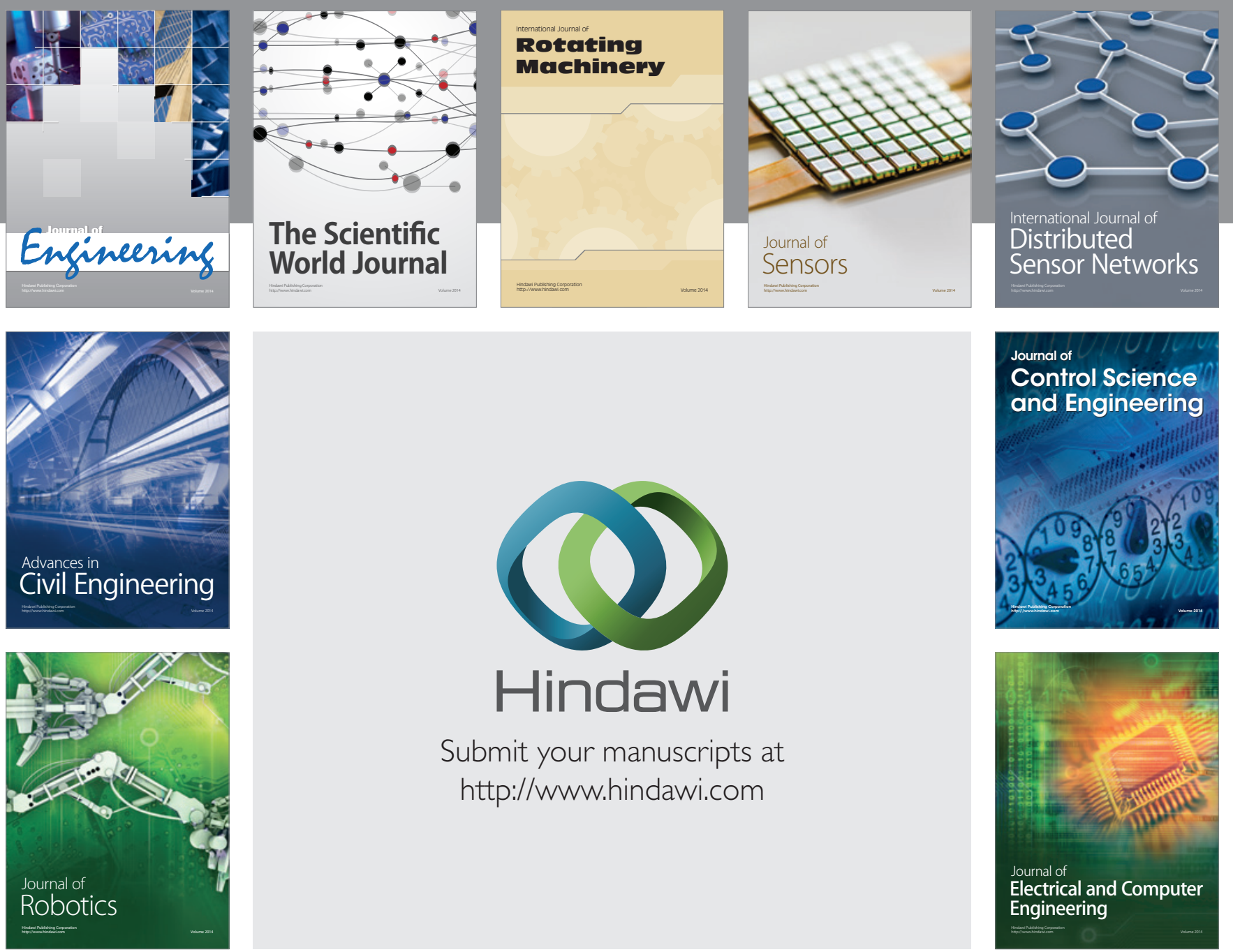

Submit your manuscripts at

http://www.hindawi.com
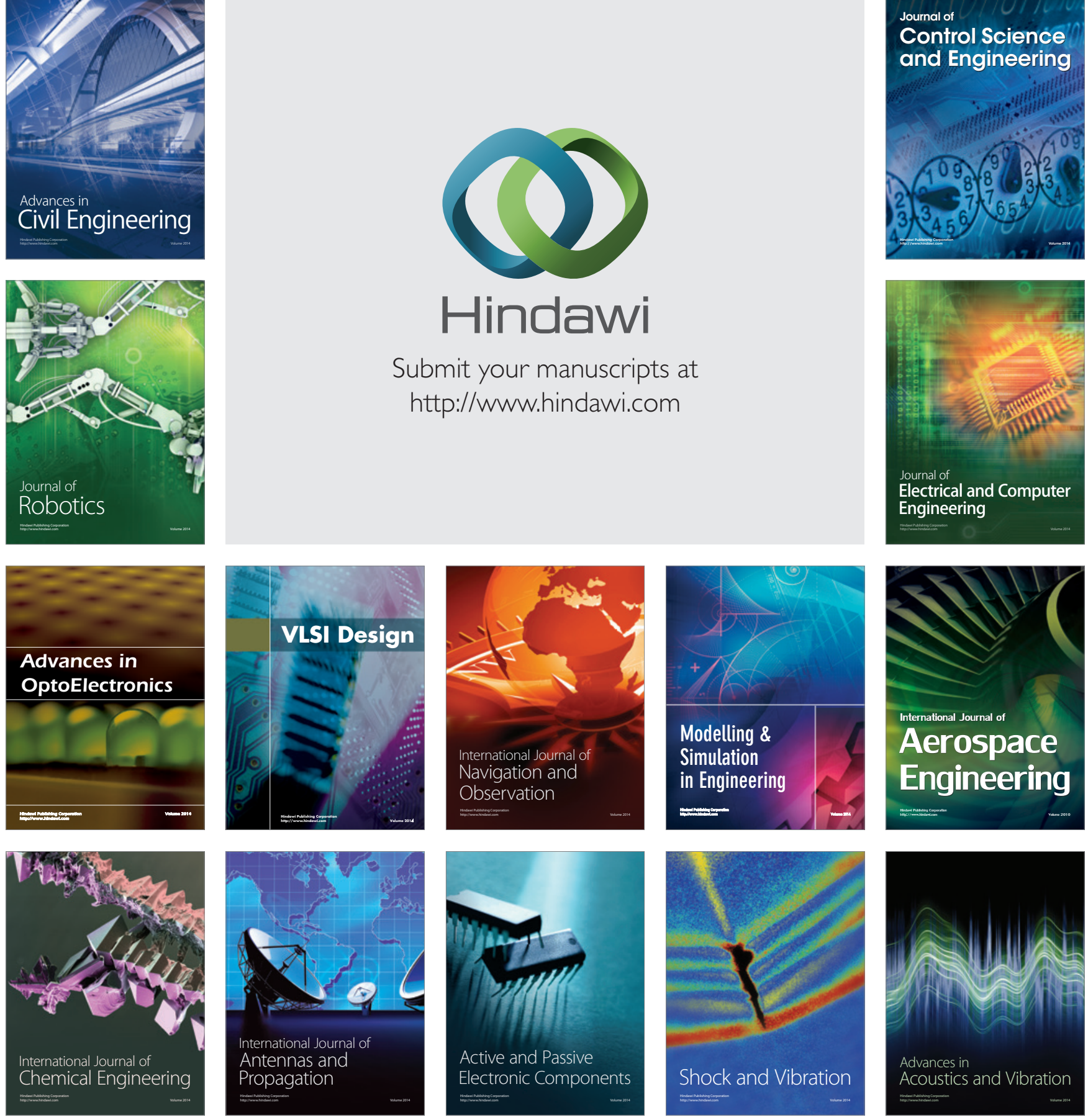